\title{
Quantum Theory of Disturbance and Delay of GPS Signals in $D$ and $E$ Atmospheric Layers: An Introduction
}

\author{
Gennady Golubkov ${ }^{1,2}$, Michael Manzhelii' ${ }^{1}$ Lev Eppelbaum³ \\ ${ }^{1}$ Semenov Institute of Chemical Physics of the Russian Academy of Sciences, Moscow, Russia \\ ${ }^{2}$ Russian Research Center at Kurchatov Institute, Moscow, Russia \\ ${ }^{3}$ Porter School of the Environment and Earth Sciences, Tel Aviv University, Tel Aviv, Israel \\ Email: kolupanovo@gmail.com, levap@post.tau.ac.il
}

How to cite this paper: Golubkov, G. Manzhelii, M. and Eppelbaum, L. (2018) Quantum Theory of Disturbance and Delay of GPS Signals in $D$ and $E$ Atmospheric Layers: An Introduction. Positioning, 9, 13-22.

https://doi.org/10.4236/pos.2018.92002

Received: April 25, 2018

Accepted: May 27, 2018

Published: May 30, 2018

Copyright $\odot 2018$ by authors and Scientific Research Publishing Inc. This work is licensed under the Creative Commons Attribution International License (CC BY 4.0).

http://creativecommons.org/licenses/by/4.0/

\begin{abstract}
GPS signals play a very important role in the modern industry, science, tourism, military and domestic operations. However, GPS signals are not free from some mistakes caused by disturbances appearing in $D$ and $E$ layers of the atmosphere. A quantum approach is proposed to the theory of propagation of a satellite GPS signal through the $D$ and $E$ layers of the atmosphere, which reduces to the problem of scattering of photons moving in the electromagnetic field of a signal in Rydberg complexes formed in a two-temperature non-equilibrium plasma. The processes of creation of additional photons as a result of stimulated emission and resonance scattering of photons are considered. It is shown that the first process leads to a direct increase in the power of the received signal, and the second to a shift in the signal carrier frequency and the time delay of its propagation. This occurs because of the scattering of the Rydberg electron by the ion core and the neutral medium molecule in the intermediate autoionization states of the composite system populated by the strong non-adiabatic coupling of electron and nuclear motions.
\end{abstract}

\section{Keywords}

GPS, $D$ and $E$ Atmospheric Layers, Rydberg Complexes, Non-Equilibrium Plasma

\section{Introduction}

The GPS system is employed now as a very important element of the global information infrastructure e.g. [1]. Free and reliable access to this system led to the development of hundreds of applications that affects every aspect of modern life. 
GPS technology is now used almost everywhere, from precise clocks and cell phones, to bulldozers, sea vessels, military applications and bank computers. It increases productivity in various sectors of the economy, including agriculture, aviation, building, defense systems, all branches of Earth's sciences, postal services, etc. The use of the GPS system saves lives, prevents traffic accidents, and promotes search and rescue operations, speeds up the delivery of emergency services and the elimination of the consequences of natural disasters. This system is vital for the next generation of the automatic transportation [2], which should help to improve vehicle safety with increasing reliability and capacity. GPS is also of high importance for the majority of scientific, environmental, technical and medical tasks: weather (climate) forecasting e.g. [3], earthquake monitoring, searching economic minerals, environmental protection, archaeological prospection, and safe driving [4]-[11]. The GPS system (space sensor net) is also crucial for any national security.

\section{Problem Formulation}

The theory of radiation, propagation and absorption of light originated in the beginning of the $21^{\text {th }}$ century and is described in detail in the courses of common and theoretical physics e.g. [12]. In our problem, we will talk about the features of the propagation of photons radiated by satellite through a resonance quantum medium in the $D$ and $E$ layers of the upper atmosphere of the Earth. Suppose that the transmitter signal is a certain group $N_{0}$ of photons sent in time $\tau_{0}$ in a certain order. The energy of a photon (here and in the future, we will use the atomic system of units $\left.\hbar=e=m_{e}=1\right)$ is defined as $E_{0}=2 \pi v_{0}$, where $v_{0}$ is the carrier frequency of the transmitter, which is assumed to be given. The total energy of the group will be $E\left(N_{0}\right)=2 \pi N_{0} v_{0}$, where $N_{0}>1$. For simplicity, we write the initial radiation power of the group (without taking into account the details of the emission of photons) as $P\left(v_{0}\right)=2 \pi N_{0}\left(v_{0} / \tau_{0}\right)$. Then the power of the radio emission flux has the form

$$
I\left(v_{0}\right)=2 \pi N_{0}\left(v_{0} / \tau_{0} S\right)
$$

where $S$ is the area of the flux.

When a satellite signal propagates through the $D$ and $E$ layers of the atmosphere, photons (moving in its electromagnetic field) are scattered on the states of orbitally degenerate Rydberg complexes $A^{* *} M$ [13], which are split by inelastic interaction with the neutral medium. The symbol $\mathrm{A}^{\star *}$ denotes the highly excited states of atmospheric molecules $\mathrm{N}_{2}, \mathrm{O}_{2}$ and $\mathrm{NO}$, and $\mathrm{M}$ includes quadrupole diatomic molecules of nitrogen and oxygen. The total wave functions of these states is superposition over the angular momenta $l$ of the weakly bound electron (for $l>3$ ) and are characterized by the angular momentum of the electron $L$ with respect to the M molecule [14]. The values of the levels shift of the $\Delta_{L}$ complex split off from the degenerate Coulomb levels due to the interaction of $\mathrm{A}^{* *}$ with the molecule of the medium $\mathrm{M}$ are noticeably greater (by an order of magnitude $n^{2}$, where $n$ is the principal quantum number) than the shifts $\Delta_{L}$ of 
the isolated Rydberg levels (for $l \leq 3$ )

$$
\Delta_{L} \sim \frac{a}{n^{4}}>\Delta_{l}
$$

where $\alpha$ is the electron scattering length on the molecule M.

Under these conditions, the influence of the neutral medium reduces to an irreversible process $l$ of mixing $A^{* *}(n, l \leq 3)+M \rightarrow A^{* *}(n, l \geq 3)+M$ with formation of the long-lived orbitally degenerate complexes $A^{* \star} M$ that are populated in a two-temperature plasma at the height of $80-110 \mathrm{~km}$ over the Earth [14]. At the lower boundary of this layer (domain), the Rydberg states are effectively quenched. The upper boundary of this layer corresponds to the restriction on the density of the medium for the mixing process $I$ [13]. The characteristic frequency of transitions $v=\Delta_{L} / 2 \pi$ in the complex $A^{\star \star} M$ (occurring without changing the principal quantum number $n$ ) in this region is of the order of 1 $\mathrm{GHz}$ (or $10^{-5} \mathrm{eV}$ ).

The strength of the satellite signal field is determined by its power and, according to Equation (1), is proportional to the number of photons $N_{0}$. Since the wavelengths of the satellite GPS signal belong to the decimeter range and are much larger than the size of the complex, all the transitions can be considered using the dipole approximation. These transitions represent an electromagnetic interaction operator with the complex in the form of $V=-\mathbf{f D}$, where $\mathbf{f}$ is the quantity of the field strength operator determined by the source power, and $\mathbf{D}$ is the dipole moment operator of the complex $A^{* *} M[12]$.

The magnitude of the propagation time delay is described by a simple formula proposed in [15]:

$$
\Delta \tau=\frac{40.3}{3 \cdot 10^{8} v^{2}} \int N d l
$$

where vis the radiofrequency emitted by the satellite (in $\mathrm{Hz}$ ), $\int N d l$ is the ionospheric total electrons content (TEC) in the signal propagation path, along the column cross-section for one $\mathrm{m}^{2}$.

This model [15] is based on the assumption that the Earth's ionosphere is a dispersive plasma medium that, strictly speaking, is valid only for the linear homogeneous media where harmonic fields independently exist. In essence, this is the simplest model of ionospheric plasma where the dispersion of the medium is associated with variations of the electron density at a fixed frequency. In real situations, we have more complex model of this phenomenon [16] [17].

The main goals of this investigation are following:

1) to establish the relationship between the observed features of the propagation of satellite signals through the atmosphere with the quantum dynamics of processes occurring within the orbitally degenerate Rydberg complexes,

2) to estimate the characteristic values of the shift and time delay of the signal in one act of interaction with the complex, which was not previously reported in the literature was considered.

It is important to note that Equation (3) is empirical and it turns out to be 
completely unsuitable for an increase in solar activity and occurrence of magnetic storms and disturbances [18] [19].

\section{Types of Transition in Interaction of Photon with the Rydberg Complex}

The description of the orbitally degenerate states of Rydberg complexes $A^{* *} M$ requires, at a first stage, the introduction of diabatic vibronic potential energy surfaces (PES) $U_{n_{v}, L}\left(R, \xi_{M}, u\right)$, where the vector $R$ is the distance between the diatomic molecules $\mathrm{A}^{\star *}$ and $\mathrm{M}, u$ is their relative velocity, and the vector $\xi_{M}$ specifies the orientation of the molecule $\mathrm{M}$ in the coordinate system associated with the molecule $\mathrm{A}^{* *}$.

Under vibronic we mean the electron-vibrational states of the Rydberg complex with the principal quantum number $n_{v}$ (depending on the vibrational quantum number $v$ of the molecular ion $A+$ ) and the moment of the weakly bounded electron $L$ relative to the molecule M. A regular method for construction of such calculation scheme is suggested in [13] [20]. A rotation of the molecule $\mathrm{M}$ can be considered classically and we assume that the direction of its axis to be fixed, averaging over the orientations at the last stage of the calculations [18] [21] [22].

In addition, we should average here the velocities, confining ourselves to the Maxwell distribution, since the main contribution is done by the domain of relative energy that is less or equal to the temperature of the neutral medium. Since the radiation processes in this formulation of the problem are described as quasi-static one, the frequency of radiation (or absorption) transitions is determined by the relation

$$
v_{v}\left(L_{i} \leftrightarrow L_{f}\right)=\frac{1}{2 \pi}\left|U_{n_{v}, L_{i}}\left(R, \xi_{M}, u\right)-U_{n_{v}, L_{f}}\left(R, \xi_{M}, u\right)\right|
$$

Therefore, all subsequent averaging over orientations and relative velocities should be performed for a given transition with $v$ frequency.

To understand the physics of the phenomenon, it suffices to confine ourselves to three types of transitions. The first concerns the intrinsic spontaneous microwave radiation of a neutral quantum medium, which is incoherent and propagates in an arbitrary direction. The dissociation of Rydberg complexes $A^{* *} M$ is not taken into account in this case, since the distribution of populations of Rydberg complexes with respect to energy levels in a nonequilibrium plasma is assumed to be stationary and is maintained by a constant flux of free electrons [14].

The second is a direct transition $N_{0} \rightarrow N_{0}+1$ to the underlying state of the complex and corresponds to stimulated emission at the carrier frequency $v_{0}$ (Svanberg, 2001). It preserves the polarization of the photons of the satellite signal and can also be considered without taking into account a pre-dissociation. This leads to the creation of additional photons and the experimentally observed increase in the power of the received GPS signal. This effect was first discovered 
in the Afraimovich's unique experiment [23], which was held at the Cornell University (USA) and has not been explained until the present time. Direct measurements have shown that the power of the receiver's received radiation at the frequency $v_{0}$ increases three-fold compared to the transmitter power on the satellite and is accompanied by signal failures at the receiver.

The third type of transition corresponds to the process of resonance scattering of a photon on a complex $A^{* *} M$, which proceeds through three successive stages. The first corresponds to stimulated absorption of a photon with $L \rightarrow L^{\prime}$ transition to an intermediate overlying $n_{v}, L^{\prime}$ is the vibronic state of the complex. The next stage is connected with the quantum dynamics within the complex itself $A^{* *} M$, where along with the discrete states, the energy spectrum of the composite system contains autoionization states of the $\mathrm{A}^{\star *}$ molecule. This is realized when the relative energy of the diatomic molecules $A^{* *}$ and $M$ in the complex $\varepsilon \sim T_{a}$ is greater than the binding energy of the electron. Physically it corresponds to the case when $n \geq\left(1 / \sqrt{2 T_{a}}\right) \geq 30$, where $T_{a}$ is the temperature of the neutral medium. A similar situation was considered earlier in [21].

The process ends by the subsequent forced transition $L^{\prime} \rightarrow L^{\prime \prime}$ to the final state $\left(N_{0}-1\right) \rightarrow N_{0}$. We note that the general scheme of resonance scattering of photons on highly excited Rydberg molecules significantly differs from the traditional scheme of the processes described in [12], which in principle cannot lead to a time delay. We also note that in the problem of resonance scattering of a Rydberg electron, the presence of predissociation channels should be taken into account when considering the dissociation of complexes for various fragments of a neutral medium independently [24]. Thus, the theory of propagation and distortion of satellite signals should include simultaneously the contributions of all three mentioned types of the radiation processes.

\section{Discussion}

For a qualitative explanation of the features of GPS signal distortion during the propagation through a $60-110 \mathrm{~km}$ layer, it suffices to confine ourselves to the case of resonance (Rayleigh) elastic scattering (when the quantum state of the Rydberg complex $A^{\star \star} M$ does not change) and analyze the resonant structure of the transition oscillator strength. The problem of the radiative transition to the ionization continuum of the Rydberg molecule (bound-free transitions) was considered in [25]. In this work, the total wave functions of the molecule are expressed in terms of the collision T-matrix elements describing the scattering of a slow electron on the ion core in the Coulomb potential and the force field of the $\mathrm{M}$ molecule [24]. Consequently, the dipole matrix element $D_{\text {if }}$ becomes a superposition of the direct and two-stage (resonant) processes, and the strength of the transition oscillator acquires a typical form of the Fano contour.

Since in our case the initial and final electronic states of the Rydberg complex $\mathrm{A}^{* *} \mathrm{M}$ are related, the frequency shift of the scattered photon $\Delta v_{0}$ and the delay time $\Delta \tau$ are formed in the intermediate second stage of the resonance process. 
This is due to the presence of a free term in the collision in the T-matrix, leading to a shift and broadening of the resonant lines [25]. They are determined by the multichannel electrostatic interaction of the Rydberg electron with the ion core $V^{e}$ and the configuration interaction $V^{C I}$ with the dissociative continuum. Moreover, for the levels related to the vibrational excited states of the $A+$ ion (located near the corresponding levels of the series $V=0$ ), the connection with the dissociative continuum increases substantially [17] due to the decrease of $I$ and the principal quantum number $n_{r}$. Behavior of the mentioned factors is of high importance since the total wave function includes a superposition of all possible vibronic states with a given energy that also contribute to the shift of the photon frequency.

For a qualitative explanation of the propagation and distortion of the GPS signal, it suffices to limiting the case when the resonant (Rayleigh) elastic scattering (when the quantum state of the Rydberg complex $A^{* \star} M$ does not change) and analyze the resonant structure of the oscillator absorption strength of the photon $F_{n_{v}},\left(L \rightarrow L^{\prime}\right)$.

Consequently, the dipole matrix element $D_{n_{v}},\left(L \rightarrow L^{\prime}\right)$ becomes a superposition of the direct and two-stage (resonant) processes acquiring a typical form of the Fano contour. For pre-dissociation states, this situation arises with the participation of discrete vibronic levels of the vibrationally excited states of the $\mathrm{A}^{+}$ion (located near the corresponding levels of the series $v=0$ ). It is caused by substantial increase of connection between the Rydberg configuration and the dissociative continuum due to the decrease of land the principal quantum number $n_{v}$, since the total wave function includes the superposition all possible vibronic states with a given energy. As a result, the strength of the strength of the transition oscillator $\left(L \rightarrow L^{\prime}\right)$ can be represented in the form

$$
F_{n_{v}}\left(L \rightarrow L^{\prime}\right)=F_{n_{v}}^{(0)}\left(L \rightarrow L^{\prime}\right)\left[\frac{\left(q_{n_{v}}+\delta_{n_{v}}\right)^{2}}{\left(1+\delta_{n_{v}}^{2}\right)}\right]
$$

where $F_{n_{v}}^{(0)}$ is the strength of the direct transition oscillator, $q_{n}$ is the profile index, $\delta_{n_{v}}=4 \pi \Delta v / \Gamma_{n_{v}}$ is the reduced shift of the carrier frequency, and $\Gamma_{n_{v}}$ is the width of the resonant level.

It is seen that the frequency shift of the scattered photon $\delta v_{0}$ and the delay time $\delta \tau$ in the intermediate state (due to the broadening of the autoionization of discrete levels) are completely determined by the strong nonadiabatic coupling of the electron and nuclear motions in the intermediate complex $\mathrm{A}^{\star \star} \mathrm{M}$ and the configurational interaction $V^{C I}$ with the dissociative continuum. The corresponding complex dipole transitions $\left(L \rightarrow L^{\prime}\right)$ are formed by an entire set of principal quantum numbers $n$ (Figure 1 ).

Curves shown in Figure 1 represent the frequency dependence of the radiation $v$ on the principal quantum number calculated at the $R=n$ distance. Lines begin from the position, which corresponds to a strong magnetic storm (with the electron density $n_{e}=10^{7} \mathrm{~cm}^{-3}$ and temperature $T_{e}=3500 \mathrm{~K}$ ). Over the 


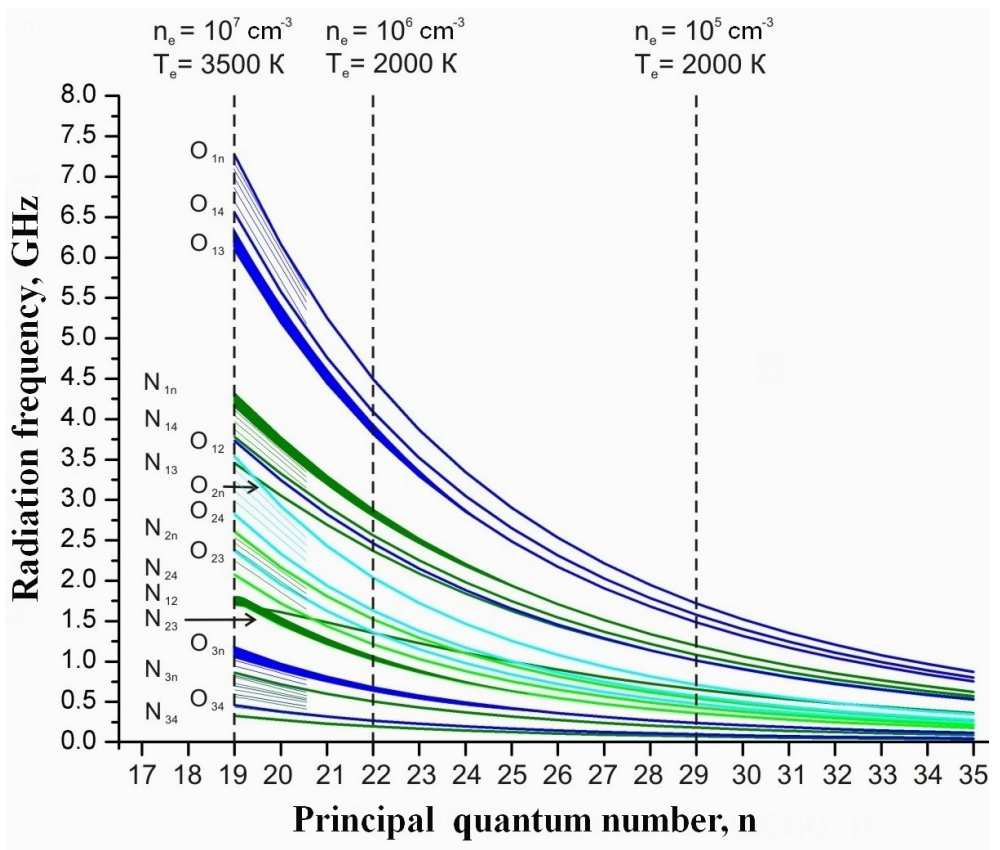

Figure 1. Lines of radiation (absorption) of quasimolecules $A^{\star \star} \mathrm{N}_{2}$ and $\mathrm{A}^{\star \star} \mathrm{O}_{2}$.

frequency range up to $10 \mathrm{GHz}$, the distribution of emission lines depending on $n$ and corresponding to $L=0-3$ contains four series of $L \rightarrow L^{\prime}$ transition lines for each molecule. These series converge as $L^{\prime}$ increases to the $L \rightarrow n$ transition limit. For $N_{2}$ and $\mathrm{O}_{2}$ molecules, these lines are denoted by $N_{L L^{\prime}}$ (marked in green) and $O_{L L^{\prime}}$ (marked in blue), respectively. The frequency shift of $N_{L n}$ and $O_{L n}$ series limits with respect to each other result in a substantial nonuniformity of the resulting emission spectrum picture.

The selection rule here has the form of ( $\Delta L= \pm k$, where $k$ is an integer), which is due to the lack of central symmetry for Rydberg molecules in the $\mathrm{A}^{* *}+$ M system [18].

Preliminary estimates for single-resonant photon scattering give the following values for the shift of the time delay frequency $\Delta \tau$. $\sim 10^{-2} \mathrm{GHz}$ or $\sim 10^{-10} \mathrm{~s}$, respectively. Consequently, the formation of observable values of the delay time when a satellite signal passes through the atmospheric layer of $60-110 \mathrm{~km}$ should occur of no less than $10^{6}$ scattering events of a group $N_{0}$ of photons. This agrees well with the previous calculations [18], since the total number of Rydberg complexes in a column with an area per receiver antenna of $10 \mathrm{~cm}^{2}$, under normal conditions of the order of $10^{7}-10^{8}$. Taking into account that the propagation velocity of a signal is much higher than the characteristic velocities of molecules inside the complexes, it sufficient to confine oneself to their quasi-static description. Then, taking the normal distribution for the Brownian motion, and taking into account that the probability of one event of a resonance scattering of a photon is $w \sim 10^{-3}$, for $10^{6}$ interactions we have $\Delta \tau \sim 10^{-7}$ s, i.e. about 100 nanoseconds.

Note that, up to now, the empirical Equation (3) is embedded in the GNSS 
positioning satellite systems for the delay time estimation. However, this expression was obtained as a result of statistical processing of the received signals under the normal atmospheric (physical-chemical) conditions. The situation changes significantly in the occurrence of magnetic storms (disturbances) when the concentration and flow of electrons increase noticeably. Attempts to describe such situations within the framework of classical electrodynamics in principle cannot lead to a delay, since it is well known and confirmed experimentally that the refractive index in the ionosphere (including plasma inhomogeneities) differs from "1.0" only in the fourth decimal place [24].

\section{Conclusions}

We suggest that the resonant multiple interaction of the GPS signal with a quantum medium in atmosphere determines the delay process: this idea is firstly proposed in the scientific literature. The development of a general theory of signal propagation and signal delay should be based on an integral version of the theory of a multichannel quantum defect (MQD) and may be divided into three stages. At the first stage, it is assumed to construct a complete adiabatic wave function of the initial state of the Rydberg complex including the vibronic states and the exchange interaction (for the case where the Rydberg molecule and the neutral molecule of the medium coincide). To solve this problem, one can use the method developed in [21] for a simple system "Rydberg atom + atom with a filled electron shell", and to modify it substantially.

At the second stage, it is necessary to develop a regular method for constructing the complete wave function of the complex taking into account the dynamics of its fragments, when the kinetic energy of their relative motion is included in the general analysis. This model also was not earlier discussed, and it proposed here for the first time. This will allow us to determine the corresponding dipole matrix elements of resonant scattering and to introduce into the theory at the third stage the power of the radiation obtained into receiver and the total delay time of the signal as a sum of the rescattering cascade along the propagation path of the signal. Simultaneously, it is necessary to develop a numerical program and calculate the total delay time of the satellite signal, depending on the main parameters of the nonequilibrium plasma (concentration, flux, and electron temperature) for various geomagnetic conditions in the ionosphere. We should note that a consistent quantum theory of the propagation of satellite signals for development of more precise model of the phenomenon must also take into account the Raman scattering of photons and the possibility of decay of $A^{\star \star} M$ complexes, which have not been analyzed in the literature. This will allow calculating the reliable values of the carrier frequency shift and to compute the delay time of the incoming radio waves.

\section{Acknowledgements}

This work was supported by the Russian Foundation for Basic Research (Grant 
16-05-00052).

\section{References}

[1] Zumberg, J.F., Heftin, M.B., Jeffersonet, D.C., et al. (1997) Precise Point Positioning for the Efficient and Robust Analysis of GPS Data from Large Networks. Journal of Geophys. Research, 102, 5005-5017. https://doi.org/10.1029/96JB03860

[2] Wang, W.J., Hsu, T.M. and Wu, T.S. (2017) The Improved Pure Pursuit Algorithm for Autonomous Driving Advanced System. IEEE 10th International Workshop on Computational Intelligence and Applications (IWCIA), Hiroshima, 11-12 November 2017, 33-38. https://doi.org/10.1109/IWCIA.2017.8203557

[3] Eppelbaum, L.V. (2013) Non-Stochastic Long-Term Prediction Model for US Tornado Level. Natural Hazards, 69, 2269-2278.

https://doi.org/10.1007/s11069-013-0787-7

[4] Kaplan, E.D. and Hegarty, C.J. (2006) Understanding GPS Principles and Applications. 2nd Edition, Artech House, Norwood.

[5] Finkelstein, M., Price, C. and Eppelbaum, L. (2012) Is the Geodynamic Process in Preparation of Strong Earthquakes Reflected in the Geomagnetic Field? Journal of Geophysics and Engineering, 9, 585-594. https://doi.org/10.1088/1742-2132/9/5/585

[6] Eppelbaum, L.V. (2011) Study of Magnetic Anomalies over Archaeological Targets in Urban Conditions. Physics and Chemistry of the Earth, 36, 1318-1330. https://doi.org/10.1016/j.pce.2011.02.005

[7] Eppelbaum, L.V. and Mishne, A.R. (2011) Unmanned Airborne Magnetic and VLFinvestigations: Effective Geophysical Methodology of the Near Future. Positioning, 2, 112-133.

[8] Eppelbaum, L.V., Katz, Y.I. and Ben-Avraham, Z. (2012) Israel-Petroleum Geology and Prospective Provinces. AAPG European Newsletter, No. 4, 4-9.

[9] Rampinini, E., Alberti, G., Fiorenza, M., Riggio, M., Sassi, R., Borges, T.O. and Coutts, A.J. (2004) Accuracy of GPS Devices for Measuring High-Intensity Running in Field-Based Team Sports. International Journal of Sports Medicine, 36, 1-6.

[10] Eppelbaum, L.V. and Katz, Y.I. (2015) Paleomagnetic Mapping in Various Areas of the Easternmost Mediterranean Based on an Integrated Geological-Geophysical Analysis. In: Eppelbaum, L., Ed., New Developments in Paleomagnetism Research, Ser: Earth Sciences in the 21st Century, Nova Science Publisher, New York, 15-52.

[11] Eppelbaum, L.V. (2016) Remote Operated Vehicles Geophysical Surveys in Air, Land (Underground) and Submarine Archaeology: General Peculiarities of Processing and Interpretation. Trans. of the $12^{\text {th }}$ EUG Meet., Geophysical Research Abstracts, Vol. 18, EGU2016-10055, Vienna, 17-22 April 2016, 1-7.

[12] Berestetskii, V.B., Lifshitz, E.M. and Pitaevskii, L.P. (1982) Quantum Electrodynamics. Pergamon Press, Oxford.

[13] Golubkov, G.V., Golubkov, M.G. and Ivanov, G.K. (2010) Rydberg States of Atoms and Molecules in a Field of Neutral Particles. In: Bychkov, V.L., Golubkov, G.V. and Nikitin, A.I., Eds., The Atmosphere and Ionosphere: Dynamics, Processes and Monitoring, Springer, New York, 1-67. https://doi.org/10.1007/978-90-481-3212-6_1

[14] Golubkov, G.V., Golubkov, M.G. and Manzhelii, M.I. (2012) Microwave Radiation in the Upper Atmosphere of the Earth during Strong Geomagnetic Disturbances. Russian Journal of Physical Chemistry B, 6, 112-127. https://doi.org/10.1134/S1990793112010186 
[15] Klobuchar, J. (1987) Ionospheric Time-Delay Algorithms for Single-Frequency GPS Users. IEEE Transactions on Aerospace and Electronic Systems, 3, 325-331. https://doi.org/10.1109/TAES.1987.310829

[16] Golubkov, G.V., Manzhelii, M.I., Berlin, A.A., Lushnikov, A.A. and Eppelbaum, L.V. (2016) Influence of D and E Atmospheric Layers on the Propagation of Radio Waves and Satellite Signals. Journal of Lasers, Optics \& Photonics, 3, 89.

[17] Golubkov, G.V., Manzhelli, M.I., Berlin, A.A., Lushnikov, A.A. and Eppelbaum, L.V. (2016) The Problems of Passive Remote Sensing of Earth Surface. Atmosphere, Ionosphere, Safety, Kaliningrad, 35-40.

[18] Golubkov, G.V., Golubkov, M.G. and Manzhelii, M.I. (2014) Rydberg States in the Atmosphere D Layer and GPS System Positioning Errors. Russian Journal of Physical Chemistry B, 8, 103-115. https://doi.org/10.1134/S1990793114010126

[19] Eppelbaum, L.V., Golubkov, G.V., Manzhelii, M.I., Berlin, A.A. and Lushnikov, A.A. (2017) Distortion of the 4.0-6.0 GHz Range Radio Signal in the E and D Atmospheric Layers. Proceedings of the World Congress on Marine Sciences, Atlanta, 16-17 November 2017, 55.

[20] Golubkov, G.V. and Ivanov, G.K. (2001) Rydberg States of Atoms and Molecules and Elementary Processes with Their Participation. URSS, Moscow. (In Russian)

[21] Golubkov, G.V., Devdariani, A.Z. and Golubkov, M.G. (2002) Collision of Rydberg Atom A with Atom B in the Ground Electronic State. Optical Potential. Journal of Experimental and Theoretical Physics, 95, 987-997.

[22] Golubkov, G.V. and Golubkov, M.G. (2014) Perturbation of Highly Excited States of an Atom by the Field of a Neutral Particle. Russian Journal of Physical Chemistry $B, \mathbf{8}, 35-44$. https://doi.org/10.1134/S1990793114010114

[23] Afraimovich, E.L., Astafieva, E.I., Berngardt, O.I., et al. (2004) Mid-Latitude Amplitude Scintillation of GPS Signals and GPS Performance Slips at the Auroral Oval Boundary. Radiophysics and Quantum Electronics, 47, 453-468. https://doi.org/10.1023/B:RAQE.0000047237.67771.bc

[24] Golubkov, G.V., Golubkov, M.G., Manzhelii, M.I. and Karpov, I.V. (2014b) Optical Quantum Properties of GPS Signal Propagation Medium-D Layer. In: Bychkov, V.L., Golubkov, G.V. and Nikitin, A.I., Eds., The Atmosphere and Ionosphere: Elementary Processes, Monitoring, and Ball Lighting, Springer, New York, 1-68.

[25] Balashov, E.M., Golubkov, G.V. and Ivanov, G.K. (1984) Radiative Transitions between the Rydberg States of Molecules. Journal of Experimental and Theoretical Physics, 59, 1188-1194. 